\title{
Direct measurement of the critical velocity above which a tuning fork generates turbulence in superfluid helium
}

\author{
V. B. Efimov • Deepak Garg • O. Kolosov • \\ P. V. E. McClintock
}

Received:September 28, 2009/ Accepted: date

\begin{abstract}
The dynamics of an electrically-driven $8 \mathrm{kHz}$ quartz tuning fork has been studied experimentally in liquid helium- 4 in the temperature range $1.3<T<4.2 \mathrm{~K}$ under the saturated vapour pressure. The fork has relatively large dimensions compared to standard $32 \mathrm{kHz}$ fork used in recent investigations. The velocity of the tip of the fork prong is measured by the indirect electromechanical equivalent method and is compared with the velocity of another $8 \mathrm{kHz}$ fork (from the same batch) determined by direct optical measurement of the oscillation amplitude through Michelson interferometry. A comparison of these results has provided absolute values for the critical velocity of the transition to the turbulent state.
\end{abstract}

Keywords quantum fluid $\cdot$ quantum turbulence $\cdot$ tuning forks $\cdot$ superfluid helium . critical velocity

PACS $67.25 . \mathrm{dk} \cdot 67.25 . \mathrm{dg} \cdot 47.27 . \mathrm{Cn} \cdot 07.60 . \mathrm{Ly}$

\section{Introduction}

Quantized vortices and their dynamics in superfluid helium-4 are often studied by analyzing the dynamics of vibrating objects, which can be used for both generation and detection of vortices. In recent years, quartz tuning forks have been widely used for this purpose. Although, used primarily as frequency standards $(32,768 \mathrm{~Hz})$ in watches, these inexpensive, robust and magnetically insensitive forks have shown [1] a promising response as probes to study the properties of low viscosity liquids. They have been exploited for studies of laminar, potential and turbulent flows in normal and superfluid helium-4 [2-4], where the onset of turbulence is defined by a threshold (critical) velocity of the flow. The measurement methods in previous studies include a bridge scheme [1] and a current measurement scheme [2], where the current is measured across a resistor

\section{B. Efimov}

Institute of Solid State Physics RAS, Chernogolovka, Russia

E-mail: victor_efimov@yahoo.co.uk

V. B. Efimov, Deepak Garg, O. Kolosov, P. V. E. McClintock

Physics Department, Lancaster University, Lancaster, LA1 4YB, UK 
(typically $1 \mathrm{k} \Omega$ ) or using a high-gain low-noise current-to-voltage $(I / V)$ convertor $[5$, $6]$ that helps to minimize signal losses due to stray capacitances.

In the first part of the paper, we will describe the electromechanical equivalence parameters for the first fork. After that the optical interferometry measurement technique will be described in detail for the second fork and a comparison will be made between the fork velocities measured electrically and optically. Finally, the force-velocity relation will be obtained for the first fork in He-II at different temperatures and the critical velocities for turbulence generation at these temperatures will be described.

\section{Experiments and results}

The size parameters of one of the prongs of the first $8 \mathrm{kHz}$ fork are $L=9.235 \mathrm{~mm}$, $W=0.399 \mathrm{~mm}, T=0.909 \mathrm{~mm}$, where $L, W, T$ represent the length, width and the thickness of each prong. The separation between the inward-facing ends of the prongs was $D=0.49 \mathrm{~mm}$. The measured resonant frequency at room temperature in vacuo (pressure below 1 mbar) was $f_{R}=8458.73 \mathrm{~Hz}$ with a $Q$-factor $\approx 10^{5}$ (the width of the resonance curve being $\Delta f=0.072 \mathrm{~Hz}$ ). The vacuum measurement was repeated at low temperatures under continuous pumping $(P<2$ mbar) of superfluid helium with the fork just above the liquid level, yielding $f_{R}=8443.9 \mathrm{~Hz}$, while the quality of the fork increased three-fold (the vacuum-helium measurement).

\subsection{Calibration of fork velocity}

The velocity calibration of the fork is done electrically by finding its electromechanical constant [2] $a$ (also called the fork constant). The theoretical value of $a$ is given by [8]

$$
a_{T}=3 d_{11} E \frac{T W}{L}
$$

where $d_{11}=2.31 \times 10^{-12} \mathrm{~m} / \mathrm{V}$ is the longitudinal piezoelectric modulus of quartz and $E=7.87 \times 10^{10} \mathrm{~N} / \mathrm{m}^{2}$ is the elasticity modulus of quartz. In our case $a_{T}=2.142 \times 10^{-5} \mathrm{C} / \mathrm{m}$.

Experimentally, the fork constant $a$ is measured by considering the fork oscillator as a series $L-C-R$ resonator. The fork is excited with an ac voltage $U=U_{0} \cos (\omega t)$ while the frequency is slowly swept through resonance. The stress due to the fork deflection induces charge proportionately, and thus the induced current $I(t)$ is proportional to the derivative of the fork deflection i.e. to the velocity.

$$
I(t)=a \frac{d x}{d t}
$$

Here the fork constant $a$ connects the mechanical properties of the tuning fork to its electrical equivalent properties. This current $I(t)$ is measured across a resistor $(\sim$ $995 \Omega$ ) and the corresponding voltage response is measured on a Stanford Research SR-830 low-noise lock-in amplifier. In this case, the electro-mechanical coefficient of the fork response may be found [9] from the equation

$$
a_{E M}=\sqrt{\frac{2 m^{*} \Delta \omega}{R}},
$$


where $m^{*}=0.2426 \rho L W T$ is the effective mass of one prong in vacuum [8] and is $2.161 \times 10^{-6} \mathrm{~kg}$ for our fork. $\Delta \omega$ is $2 \pi$ times the width of the resonance curve $I(f)$ and $R$ is the electrical resistance of the fork and is determined by the current amplitude at resonance for a given drive for which the response is linear. The $8 \mathrm{kHz}$ fork at room temperature in vacuo gives $R=19.902 \mathrm{k} \Omega$ with a corresponding width $\Delta f=0.072 \mathrm{~Hz}$. The calculated value of the fork constant $a_{E M}$ was $0.97 \times 10^{-5} \mathrm{C} / \mathrm{m}$ which amounts to $45 \%$ of the theoretical value given by equation (1). The experimental value of $a$ measured in air is within $2 \%$ of the value measured in vacuum. This is because of the low density of the air, the prong mass in air $m_{\text {air }}{ }^{*}$ being very close to the vacuum mass in equation 3 . The second reason is due to the experimental fact that the value of $\Delta f / R$ is constant for a given drive irrespective of the medium.

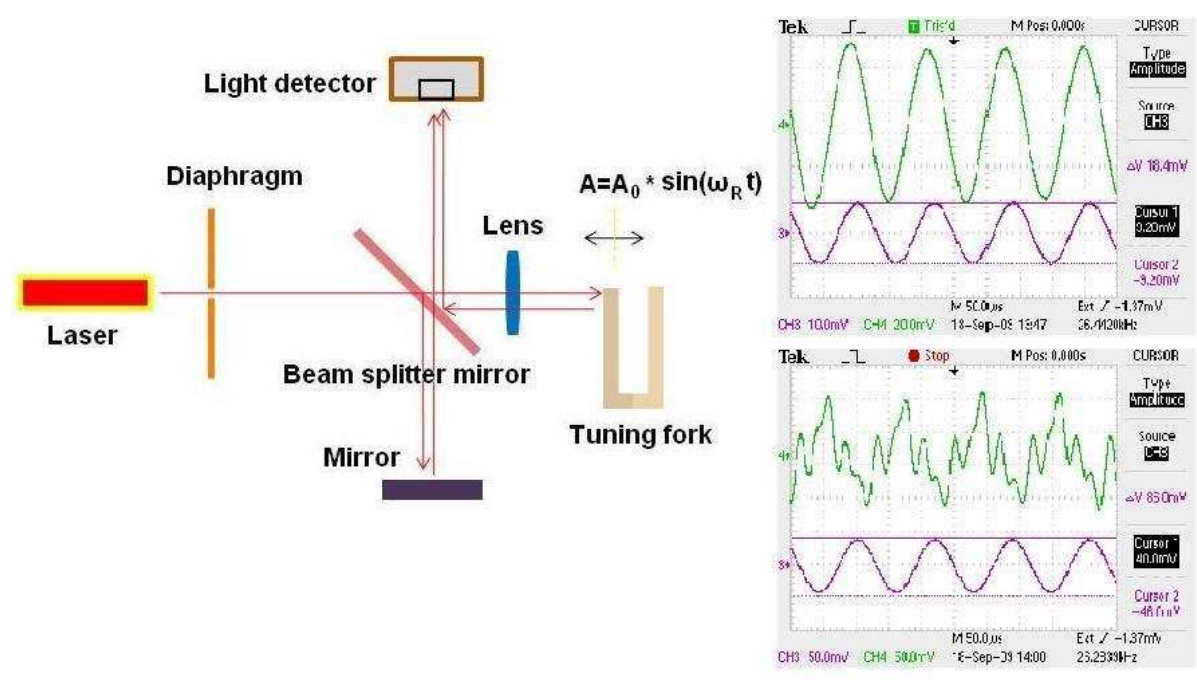

Fig. 1 (color online) Left: schematic diagram of the Michelson interferometer arrangement. Right: oscilloscope traces of interferometric signals (upper/green curves) for excitations (lower/red curves) of $U_{G}=6.5 \mathrm{mV}_{\mathrm{RMS}}$ (top) and $U_{G}=28 \mathrm{mV}_{\mathrm{RMS}}$ (bottom) at room temperature.

\subsection{Michelson interferometry}

The second $8 \mathrm{kHz}$ fork used for optical measurements has fork constant $a_{E M}$ within $2-3 \%$ of the first fork from the same batch. The direct measurement of the fork velocity driven at resonance $(8.45 \mathrm{kHz}$ in air) was determined by use of a conventional Michelson interferometry scheme at room temperature and atmospheric pressure, as illustrated schematically in Fig.1. The visible light beam from a semiconductor laser $\left(\lambda_{L}=670 \mathrm{~nm}\right)$ was split into two beams by a non-polarising beam splitter. A beam reflected from the end of the leg of the tuning fork, vibrating at the resonant frequency at a position $A(t)=A_{0} \sin \left(\omega_{R} t\right)$ interferes with another beam, reflected from the fixed mirror. The spot size of the beam on the fork leg was $\sim 0.2 \mathrm{~mm}$ and it was at a distance 
$\sim 0.9 \mathrm{~mm}$ from the tip of the leg. The amplitude of the interfering waves is proportional to sum of both waves

$$
S=B_{1} e^{i \omega t}+B_{2} e^{i\left(\omega t+\varphi_{0}+A(t)\right)}=B_{1} e^{i \omega t} \times\left(1+B_{2} / B_{1} \times e^{i\left(\varphi_{0}+A(t)\right)}\right)
$$

The interference intensity is recorded by a photodiode. Mathematically this signal is proportional to $U \sim S \times S^{*}$ and is given by

$$
U \sim 1+B^{2}+B \times e^{i\left(\varphi_{0}+A(t)\right)}+B \times e^{-i\left(\varphi_{0}+A(t)\right)}=1+B^{2}+2 \times B \times \cos \left(\varphi_{0}+A(t)\right)
$$

The alternating traces of this recorded signal $U_{a c} \sim 2 \times B \times \cos \left(\varphi_{0}+A(t)\right)$ are synchronized with the fork excitation signal shown on the right side of the Fig. 1 . The maximum recording AC signal corresponds to the phase of $\varphi_{0}=\pi / 2($ or $=(n+1 / 2) \pi)$, which was adjusted by change of the distance to the mirror, and the amplitude of the tuning fork oscillation equal to

$$
2 \times 2 \times A_{0}=\lambda_{L} / 2
$$

as shown in the upper-right part of the figure. As the drive amplitude to the fork is increased, the optical signal at first increases too, until it attains a maximum value at a drive corresponding to $A_{0}=\lambda_{L} / 8$ condition. In the present case, this condition was reached when the drive to the fork was $6.5 \mathrm{mV}_{\mathrm{RMS}}$. At higher drives the fraction of higher harmonics increases in the optical signal, meaning multiple interference maxima and minima within a single period of fork motion. For example, the lower picture shows the optical signal obtained for $28 \mathrm{mV}_{\mathrm{RMS}}$ drive to the fork and 4 peaks can be seen clearly. The 4 th peak started to appear when the fork drive was $26 \mathrm{mV}_{\mathrm{RMS}}$ which further validates $6.5 \mathrm{mV}_{\mathrm{RMS}}$ drive for the maximum optical signal. The fork amplitude at this drive would have been $A_{0}=\lambda_{L} / 8=670 / 8 \approx 84 \mathrm{~nm}$. So the optical velocity $V_{o}$ of the fork at resonance for $U_{G}=6.5 \mathrm{mV}_{\mathrm{RMS}}$ drive can be estimated as $v=A_{0} \times \omega_{R}=8.4 \times 10^{-8} \times 8.45 \times 10^{3} \times 6.28 \approx 4.5 \times 10^{-3} \mathrm{~m} / \mathrm{s}$ or $4.5 \mathrm{~mm} / \mathrm{s}$ within $20 \%$ experimental error. The maximum velocity of the tip of the fork prong is $\sim 10 \%$ higher, $v_{\max }=4.9 \mathrm{~mm} / \mathrm{s}$.

For $U_{G}=6.5 \mathrm{mV}_{\mathrm{RMS}}$ fork gives a current response of $j=32 \mathrm{nA} \mathrm{RMS}$ at resonance under similar conditions (at room temperature and air pressure). This current response corresponds to an electrical velocity (from equation 2) of the fork $v_{E M}=4.7 \mathrm{~mm} / \mathrm{s}$.

This suggests that the electrical velocity is within $5-10 \%$ of the optical velocity and hence both are equal within the experimental error. We used the fork constant $a=0.92 \times 10^{-5} \mathrm{C} / \mathrm{m}$ obtained from optical measurements.

\subsection{Fork oscillation in liquid helium}

The first fork was used for measurements in liquid helium. The force equivalent to the drive applied to the fork is driven is [2]

$$
F_{0}=(a / 2) U_{0}
$$

Some results are shown in Fig. 2. The right scale corresponds to the real (optical) maximum velocities (at the end of a prong).

At low excitation the fork response and corresponding velocity are proportional to the driving force. Higher excitation leads to the appearance of some additional mechanism of energy dissipation and to a deviation of the fork response (and its velocity) 


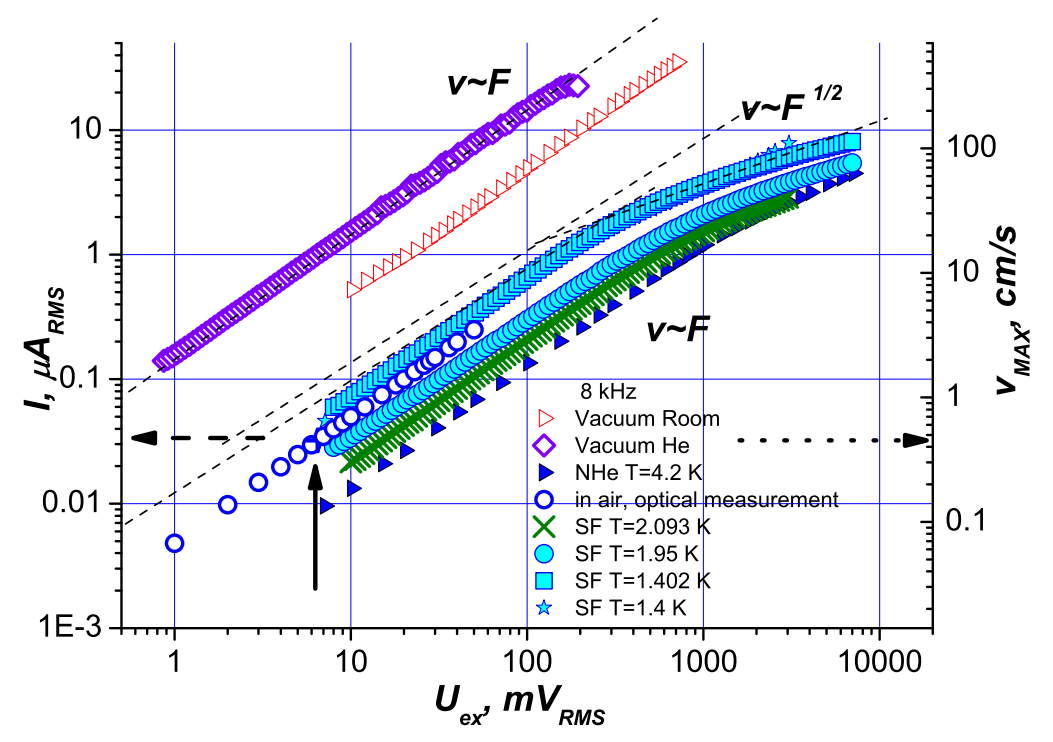

Fig. 2 (color online) The electrical response of the fork in air, in vacuum at room temperature, and in liquid helium at different temperatures. The upper curve (violet rhombuses) was obtained for low pressure of helium gas just above the superfluid helium surface (see text). The (red) open triangles represent measurement in vacuum at room temperature. The filled (blue) triangles were for He I at $T=4.2 \mathrm{~K}$. The (green) crosses and (light-blue) circles, squares and stars are results for superfluid helium at at different temperatures. $P=\mathrm{SVP}$. The response in air (blue open circles) was recorded during the optical measurements. The vertical arrow indicates the excitation at the first optical maximum. The left ordinate axis shows the fork response and the right one shows the optical prong velocity.

from linear dependence. Note that such deviations were not seen in vacuum (Fig. 2); they are usually attributed to the transition to turbulence above a critical velocity [10]. We estimated the deviation of the applied force (and counteracting fluid forces) by subtracting the linear dependence at low excitation.

$$
F_{\text {add }}=F(v)-C \times v
$$

The results are shown in Fig. 3. The fluid friction force increases very rapidly after the critical velocity $v_{c}$ is attained. We fitted the deviation by power law $F_{\text {add }}=C_{2} \times(v-$ $\left.v_{c}\right)^{N}$ at different temperatures, leading to the results presented in table 1.

In conclusion, it appears that the onset of turbulence in He II results in an additional force that rises faster than $F \propto v^{2}$. The critical velocity of $v_{c} \approx 10 \mathrm{~cm} / \mathrm{s}$ is similar to the values found in previous work. The absolute maximum velocity of the prong vibration is adequately estimated from the electro-mechanical model based on the actual lengths of the fork legs and electrical contacts.

Acknowledgements We are grateful to Ian Bradley, Rich Haley, George Pickett and Viktor Tsepelin for helpful discussions, and to Bruno Studer for providing samples of tuning forks. Special thanks are due to W. F. Vinen for his interest in the work, to Tony Guénault and Ladek Skrbek for useful remarks, and to N. Vasilev for help with the experiments. The research 


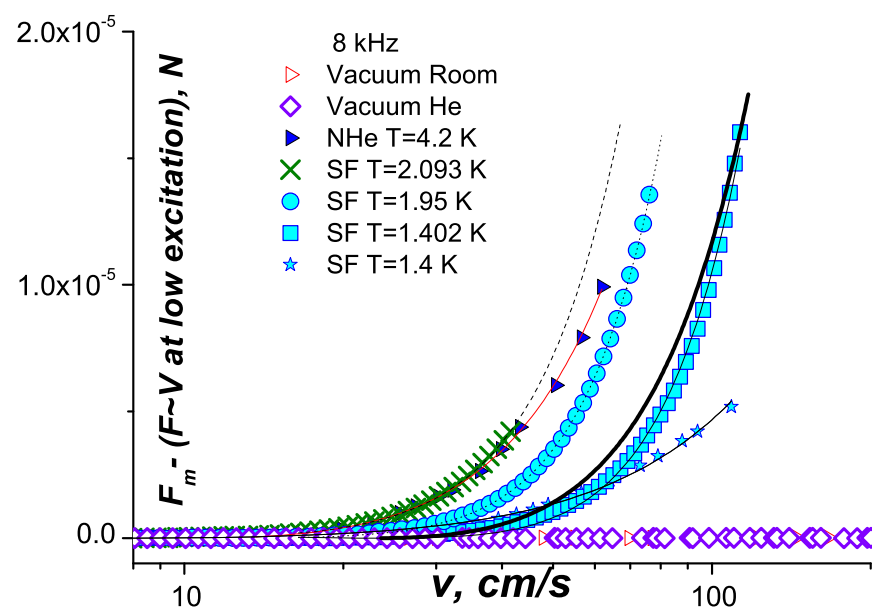

Fig. 3 (color online) The deviation from linear behavior of the fluid force resisting motion of the fork prongs $(F(v)-c \times v$, where $c \times v$ represents the linear fitting of experimental results at low excitation). The symbols are the same as for Fig.2. The thin and dashed lines represent fits to a power law with the parameters given in table 1 . The full black line represents a square-law dependence $F-F_{l i n} \propto\left(v-v_{c}\right)^{2}$ fitted to the data at $T=1.402 \mathrm{~K}$.

Table 1 The results of fitting of additional force by power law

\begin{tabular}{|c|c|c|c|}
\hline Temperature (K) & $v_{c}, \mathrm{~cm} / \mathrm{s}$ & $N$ & $C_{2}$, units $\times 10^{-9}$ \\
\hline $\mathrm{HeI}$ & 9.93 & 1.87 & 11.3 \\
\hline 2.09 & 5.54 & 2.5 & 0.56 \\
\hline 1.95 & 9.78 & 2.7 & 0.17 \\
\hline 1.402 & 21.8 & 2.43 & 0.3 \\
\hline 1.4 & 7.88 & 1.6 & 9.2 \\
\hline
\end{tabular}

was supported by the Engineering and Physical Science Research Council (U.K.) and by the Russian Academy of Sciences under the grant "Quantum physics of condensed matter".

\section{References}

1. D. Clubb, et al, J. Low Temp. Phys. 136(1/2), 1 (2004)

2. R. Blaauwgeers, et al, J. Low Temp. Phys. 146(5/6), 537 (2007)

3. G. Sheshin, et al, Low Temp. Phys. 34(11), 875 (2008)

4. M. Blazkova, et al, Phys. Rev. B 79(5), 054522 (2009)

5. O.V. Kolosov, et al, Integrated circuitry for controlling analysis of a fluid. USA Patent 7,158,897; 2 January 2007

6. P. Skyba and S. Holt, Design and construction of this convertor - to be published (2009)

7. M. Blazkova, D. Schmoranzer, L. Skrbek, Phys. Rev. E 75(2), 025302 (2007)

8. K. Karrai and R.D. Grober, Tip-sample distance control for near-field scanning optical microscopes, in Near-Field Optics, M. A. Paesler and P. T. Moyer (ed.), Proc. SPIE 2535, $69(1995)$

9. J. Rychen, et al, Rev. Sci. Instr 71, 1695 (2000)

10. W.F. Vinen, L. Skrbek, H.A. Nichol, J. Low Temp. Phys. 135(5/6), 423 (2004) 